\title{
On the Non-integrability of a Class of Hamiltonian Cosmological Models
}

\author{
L. A. A. Coelho ${ }^{1}$, J. E. F. Skea ${ }^{1}$, and T. J. Stuchi ${ }^{2}$ \\ 1 Universidade do Estado do Rio de Janeiro, IF-UERJ, \\ Rua São Francisco Xavier 524, Maracanã, 20550-900, Rio de Janeiro, RJ, Brazil \\ ${ }^{2}$ Universidade Federal do Rio de Janeiro, IF-UFRJ, Av. Brigadeiro Trompowski, sn ${ }^{o}$, \\ CT, Bloco A, $3^{\circ}$ andar, Cidade Universitária, 21941-590, Rio de Janeiro, RJ, Brazil
}

(Received on 14 October, 2005)

\begin{abstract}
The method of Morales and Ramis determines whether a given Hamiltonian system is non-integrable. We apply this method to Friedmann Robertson Walker models with a self-interacting scalar field and cosmological constant. It is shown that, with the exception of a set of measure zero, these models are non-integrable. Our results complement those of Helmi and Vucetich who used the Painlevé property to find integrable models within this class.
\end{abstract}

In recent years the search for non-integrablility criteria for Hamiltonian systems based upon the behaviour of the solutions in the complex domain has acquired more relevance. In this work we use a connection between two differents concepts of integrability: the complete integrability of complex analytical Hamiltonian systems (given by the theorem of Liouville) and the integrability of complex analytical linear ordinary differential equations (LODEs) in terms of Liouvillian functions. By Liouvillian function we mean a function which can be written as a combination of algebraic functions (solution of a polynomial equations), exponentials, their indefinite integrals, exponentials of integrals or logarithms. Since we are working in the field of complex functions, this definition includes all the usual elementary functions such as sin, cos, $\arctan$.

For a Hamiltonian system, $X_{H}$, these concepts of integrability are connected via the normal variational equation (NVE) of $X_{H}$, which may be a LODE, which describes the evolution of perturbations normal to an invariant plane of the system. The basic idea is that if the flow of the Hamiltonian system has a regular behaviour (is integrable), then the linearized flow along a particular integral curve given by the variational equation (NVE) must also be regular (integrable). Conversely if the linearized flow is non-integrable the system as a whole will be non-integrable.

must

In studying the integrability of the Friedmann-RobertsonWalker (FRW) cosmological model we use a unified and systematic approach:

1. Select a particular solution (or invariant plane).

2. Write the variational equations and the NVE.

3. Check if the solutions of the NVE are Liouvillian functions.

To decide step 3 we use a brief form of Kovacic's algorithm [1]

The dynamics of the FRW cosmological model filled with a comformally coupled scalar massive field has been studied in many papers, but none of then has put an end about the integrability of the system. Using Kovacic's algorithm to decide whether the NVEs are (or not) integrable, and the connection betweent non-integrability of the NVEs and non-integrability of the Hamiltonian system we can say with certainty that the problem is, in general, non-integrable.

\section{KOVACIC'S ALGORITHM}

Kovacic's algorithm gives us a procedure for computing the Liouvillian solution of a second order differential equation, when such a solution exists. Reciprocally, if the differential equation is non-integrable in terms of Liouvillian functions, the algorithm does not terminate. In the version used of the algorithm we essentially follow [2-4].

Given a second order LODE with coefficients in $\mathbb{C}(x)$, it is a classical fact that the ODE can be transformed to the so-called reduced invariant form

$$
\xi^{\prime \prime}-g \xi=0
$$

with $g=g(x) \in \mathbb{C}(x)$.

We can also use a well-known transformation to a Riccati equation, by the change of variables $v=\xi^{\prime} / \xi$ :

$$
v^{\prime}=g+v^{2}
$$

Then the differential equation (1) is integrable, if and only if, the equation (2) has an algebraic solution (solves a polynomial).

The key point of the algorithm is that the degree of the polynomial belongs to the set $L=\{1,2,4,6,12\}$. The algorithm has three steps: the first step is the determination of the set $L$; the two others steps are devoted to determining the existence and construction of the minimal polynomial.

If the algorithm does not work (ie, equation (2) has no algebraic solution) then equation (1) has no solution in terms of Liouvillian functions.

\section{MORALES-RAMIS THEOREM}

The Morales-Ramis theorem is a non-itegrability criterion, i.e. it says when a Hamiltonian system is non-integrable and can be read this way:

Theorem: If there are $n$ first integrals of $X_{H}$ which are meromorphic and in involution, then the EVN has Liouvillian solutions. 


\section{APPLICATION}

We use the conformal form for the metric in the FRW cosmological model

$$
d s^{2}=a^{2}(\eta)\left[-d \eta^{2}+\frac{1}{1-k r^{2}} d r^{2}+r^{2} d \Omega^{2}\right]
$$

where $a(\eta)$ is the scale factor and $k=0, \pm 1$.

With a conformally coupled self-interacting scalar field, the system can be described in terms of the Hamiltonian

$$
H=\frac{1}{2}\left[-\left(p_{a}^{2}+k a^{2}\right)+\left(p_{\phi}^{2}+k \phi^{2}\right)+m^{2} a^{2} \phi^{2}+\frac{\lambda}{2} \phi^{4}+\frac{\Lambda}{2} a^{4}\right]
$$

where $\eta$ is the conformal time, $a$ is the scale factor of the universe, $\Lambda$ the cosmological constant, and $\phi$ is the scalar field of mass $m$ and self-coupling constant $\lambda$.

The system has an invariant plane $p_{a}=a=0$, for which we obtain the normal variational equations

$$
\frac{d^{2} \delta a}{d t^{2}}=\left(-k+m^{2} \phi^{2}\right) \delta a .
$$

Changing the independent variable to $\phi$ and calling $\delta a=x$, we have the equation

$$
\frac{d^{2} x}{d \phi^{2}}+\frac{2\left(k+\lambda \phi^{2}\right)}{\phi\left(2 k+\lambda \phi^{2}\right)} \frac{d x}{d \phi}+\frac{2\left(-k+m^{2} \phi^{2}\right)}{\phi^{2}\left(2 k+\lambda \phi^{2}\right)} x=0 .
$$

This is a linear homogeneous equation with coefficients which are rational functions, of the type

$$
\frac{d^{2} y}{d x^{2}}+b(x) \frac{d y}{d x}+c(x) y=0
$$

suitable for the application of Kovacic's algorithm. Defining $r(x)=\frac{1}{2} b^{\prime}(\phi)+\frac{1}{4} b(\phi)^{2}-c(\phi) \in \mathbf{C}(x)$.

we transform this into the reduced invariant form,

$$
\ddot{y}=r(x) y
$$

with

$$
r(\phi)=\frac{\left(3 k^{2}+3 k \lambda \phi^{2}-4 m^{2} \phi^{2} k-2 m^{2} \phi^{4} \lambda\right)}{\phi^{2}\left(2 k+\lambda \phi^{2}\right)^{2}} .
$$

If we now apply Kovacic's algorithm to (9) we find that the equation is non-integrable, except for the possibly integrable cases

$$
\Lambda=-\frac{2 m^{2}}{(n+1)(n+2)}, \quad n \in \mathbb{N} .
$$

Applying the same method to the invariant plane $\left(p_{\phi}=\phi=0\right)$ we obtain a similar result: that the NVE is, in general nonintegrable except, possibly, when

$$
\lambda=-\frac{2 m^{2}}{(n+1)(n+2)}, \quad n \in \mathbb{N} .
$$

In summary, the Theorem of Morales-Ramis tells us that all these models are non-integrable with the possible exceptions when

$$
\Lambda=\lambda=-\frac{2 m^{2}}{(n+1)(n+2)}, \quad n \in \mathbb{N}
$$

\section{NUMERICAL RESULTS}

In the section we show two Poincaré sections: one for a non-integrable combination and one in the relation (12). For the non-integrable one we choose the values $\lambda=\Lambda=0$ and $m \neq 0$, and for the possibly integrable case we choose $\Lambda=$ $\lambda=-1$ and $m=1$. this figures are respectively showed below

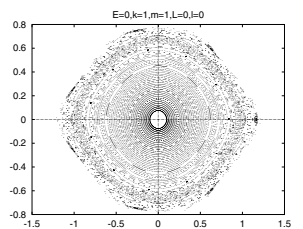

FIG. 1: Poincaré section in $p_{a} \times$ a for $\Lambda=\lambda=0$ and $m \neq 0$.

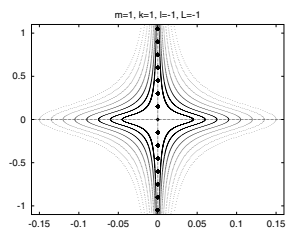

FIG. 2: Poincaré section in $p_{a} \times$ a for $\Lambda=\lambda=-1$
As can be seen from figure (1), in the first case we have behaviour typical of a non-integrable system, with broken tori and island formation. On the other hand figure (2) has regular curves around an axis filled with periodic orbits. Besides $\lambda=\Lambda=-m^{2} / 3$ (which is similar to figure (2)) the Poincaré sections of the other cases have behaviour similar to figure (1).
[1] J. J. Kovacic. J. Symb. Comput. 2, 3 (1986).

[2] J. Morales-Ruiz, Differential Galois Theory and Nonintegrability of Hamiltonian Systems (Progress in Math., Birkhäuser, 1999).

[3] A. Duval and M. Loday-Richaud. AAECC 3, 211 (1992).
[4] L. A. A. Coelho, Sobre a Integrabilidade de Sistemas Hamiltonianos Provenientes de Modelos Cosmologicos, M.Sc. thesis, UERJ, 2005. 